m10.003

\section{X-ray fluorescence holographic study on a single-crystal thin film of a rewritable optical media}

\author{
S. Hosokawa, K. Hayashi, ${ }^{\text {a }}$ N. Happo, ${ }^{\text {b }}$ and T. Ozaki \\ Hiroshima Institute of Technology, ${ }^{a}$ Tohoku University, ${ }^{b}$ Hiroshima City \\ University, Japan
}

\section{Keywords: holography, three-dimensional image, order-disorder phase transition}

In recent days, rewritable optical media DVD-RAM is widely used for recording massive data or movies. It is well-known that this recording is governed by a crystalline-amorphous transition of thin films, such as $\mathrm{Ge}_{2} \mathrm{Sb}_{2} \mathrm{Te}_{5}$. It was, however, difficult to understand the very fast recording and erasing mechanism by a normal idea of laser-induced melting and re-crystallizing process, and the real mechanism was mysterious. Recently, Kolobov et al. carried out an XAFS study on $\mathrm{Ge}_{2} \mathrm{Sb}_{2} \mathrm{Te}_{5}$ thin film [1], and found that the crystal thin film does not possess a hexagonal structure as in the bulk, but more likely consists of highly distorted rocksalt structure. The laser-induced amorphization is concluded to be due to an umbrella-flip motion of Ge atoms from an octahedral position in crystal into a tetrahedral position. Since structural information obtained from XAFS measurements is limited to be a direction-averaged pair distribution function, another probe is necessary to confirm the above unique model. X-ray fluorescence holography (XFH) is a technique that allows one to obtain a three-dimensional image around a specific element [2,3]. We have measured XFH on $\mathrm{Ge}_{2} \mathrm{Sb}_{2} \mathrm{Te}_{5}$ single-crystal thin film at the beamline BL37XU of the SPring-8. The sample with about 2 microns thickness was placed on a two-axes table of a diffractometer installed at the beamline. The Ge K $\alpha$ fluorescence $\mathrm{X}$-rays were collected using an avalanche photodiode detector with a cylindrical graphitecrystal energy-analyzer. The XFH data were recorded at several incident energies of $22.0-27.0 \mathrm{keV}$ in steps of $0.5 \mathrm{keV}$. The measurements were performed by rotating two axes, $0^{\circ} \leqslant \phi \leqslant$ $360^{\circ}$ in steps of about $0.3^{\circ}$ and $0^{\circ} \leqslant \phi \leqslant 70^{\circ}$ in steps of $0.5^{\circ}$, of the sample table, and detecting small intensity changes with angles. From the holographic patterns with the different incident energies, a three-dimensional atomic configuration image was constructed using Barton's algorithm. The obtained image clearly reveals that the $\mathrm{Ge}_{2} \mathrm{Sb}_{2} \mathrm{Te}_{5}$ single-crystal thin film does not have a hexagonal structure around the Ge atoms. The detailed analysis is now in progress.

[1] A. V. Kolobov, et al., Nature Materials 3, 703 (2004).

[2] K. Hayashi, J. Jpn. Soc. Syn. Rad. Res. 15, 267 (2002).

[3] K. Hayashi et al., Nucl. Instrum. Met. Phys. Res. B 196, 180 (2002).

\section{m10.004}

\section{Phasing in the Home Laboratory}

Richard Tyrrell, Joseph D. Ferrara, Cheng Yang, Robert Bolotovsky, James W. Pflugrath

Rigaku Americas Corp., The Woodlands, TX, 77381

\section{Keywords: SAD, phasing, chromium}

Many examples of S-SAD and Se-SAD phasing have been reported with diffraction data collected using copper radiation (1.54 $\AA$ ) or radiation at the selenium K absorption edge (0.98 $\AA$ ). With recent advances in X-ray technology, chromium radiation $(2.29 \AA)$ is now available for in-house data collection and appears to be ideally suited for measuring anomalous signals from weak anomalous scatterers such as sulfur, selenium, calcium and other atoms commonly found in protein crystals. The results of a number of successful SAD experiments using $\mathrm{Cr}$ radiation have been published by several groups including our own.

With the addition of Cr radiation to the crystallographer's toolkit, in-house X-ray sources can provide at least two routinely useful wavelength options for macromolecular crystallography. This report also discusses the results of phasing by combining diffraction data collected using both $\mathrm{Cu}$ and $\mathrm{Cr}$ radiation sources. Finally, we report the results of data collection with a new imaging plate detector (R-AXIS HR) designed specifically for use with $\mathrm{Cr}$ radiation. This new detector allows the collection of data suitable for both phasing and refinement with $\mathrm{Cr}$ radiation from a single crystal in a single, simple diffraction experiment. 